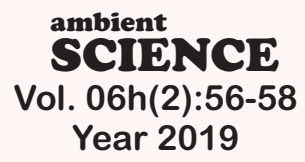

\title{
Comparative Evaluation of Aesthetic Outcomes of Rhinoplasty in Patients with Thick Nasal Skin between Type III Septal Extension Graft and Conventional Surgery
}

\section{Abdolazim Ghalambor, Kamran Babaei, Ali Mozafari*}

Department of Plastic and Reconstructive Surgery, Ahvaz Jundishapur University of Medical Sciences, Ahvaz, Iran

Study Area: Ahvaz, Iran

Coordinates: $31^{\circ} 19^{\prime} 13^{\prime \prime} \mathrm{N} ; 48^{\circ} 40^{\prime} 09^{\prime \prime E}$

Key words: Nose projection, Nose rotation, Columellar-labial angles, TP: LN

Ethical Code: The present study was approved by the local ethics committee of Ahvaz Jundishapur University of Medical Sciences (Code: IR.AJUMS.REC.1398.163) as a randomized prospective interventional study on patients with a thick skin of the candidate for surgical treatment of septorhinoplasty in theyear 2018.

\section{Abstract}

Thick nasal skin leads to projection reduction, rotation, and no proper formation of the nasal tip in the nasal plastic surgery. This study aimed to compare the aesthetic outcomes of rhinoplasty in patients with thick nasal skin between type III septal extension graft (SEG) and conventional septorhinoplasty technique. Subjects were randomly divided into two equal groups using a block size of four. The first group underwent conventional septorhinoplasty and the second group underwent SEG technique. All SEG surgeries were performed by the same surgeon and the treatment outcomes were evaluated at 3 rd and 9th months after the surgery and compared within and between groups. The outcomes were based on nasofacial angle, Tip Projection to Length of Nose (TP: LN) ratio, nasolabial angle, and nasocolumellar angle. At 3 rd and 9 th mon. of post-surgery assessments, the SEG group showed reduced (statistically) nasofacial angle, while increased nasolabial and columellar-labial angles while compared with the conventional group. Naso-columellar angle showed no significant difference after 3 mon. surgery between the two groups, but after 9 mon. surgery it was significantly higher in the SEG group. The mean TP: LN ratio, after 9 mon. surgery showed no significant change in conventional surgery, whereas increased after the SEG group.

some studies have reported good outcomes, but the others failed. Furthermore, some studies have even highlighted the risk of nasal necrosis as a consequence of conventional surgery (Katira \& Guyuron, 2015). The change in the nasal tip location mainly depends on the degree of prognosis or nasal tip markup change (Khorasani et al., 2010). It seems that due to the high weight of the skin in comparison with those with thin skin, these values (projection and rotation) change after the operation, thus the use of more precise methods with constant cartilage involvement can be effective to reduce these changes. Septal Extension Graft technique was first used for short nasal subjects to increase nasal length, and in recent years, by changing the location of these cartilages, other applications such as increased rotation, projection, and nasal type were used (Wulkan, 2015; Kim et al., 2014). Due to the above problems in subjects (persons) with thick nasal skin, we decided to know the effect of septal extension graft on improving the post- 
operative results of people with thick skin.

\section{Methodology:}

Subjects (patients) were divided into two equal groups $(n=12)$ by blocking four-digit numbers. The first group underwent surgery with conventional septorhinoplasty technique in patients with thick skin whereas the second group underwent the SEG technique (batten type). The septal augmentation in studied subjects was type III, with the lower end of the septum extending to the anterior portion. As per Kim et al. (2014) by placing this cartilage in the anterior part with an appropriate angle at the tip, it is possible to increase the nose rotation and projection, and since this graft is tight with attachment to the nasal septum, it is useful to restore the nasal tip with long-term use.

To determine the thickness of pinched skin in patients, preoperative calipers were used in the supratip area and the measurements were carried out by calipers during operation. After explaining the surgical technique and postoperative outcomes and goals of the study, informed consents were obtained from all patients. All SEG surgeries were performed by the same surgeon and the results of the treatment were compared after 3 and 9 months of surgery between two groups.

The evaluated parameters were nasofacial angle and Tip Projection to Length of Nose (TP:LN) ratio for the prognostic examination, nasolabial angle, nasocolumellar angle and columellar-lobular angle for examining the rotation of the nasal tip. Patients with severe septal deviation in the pre and postoperative examination and any problems that did not make it possible for the patient to perform SEG or conventional surgery were excluded.

\section{Result:}

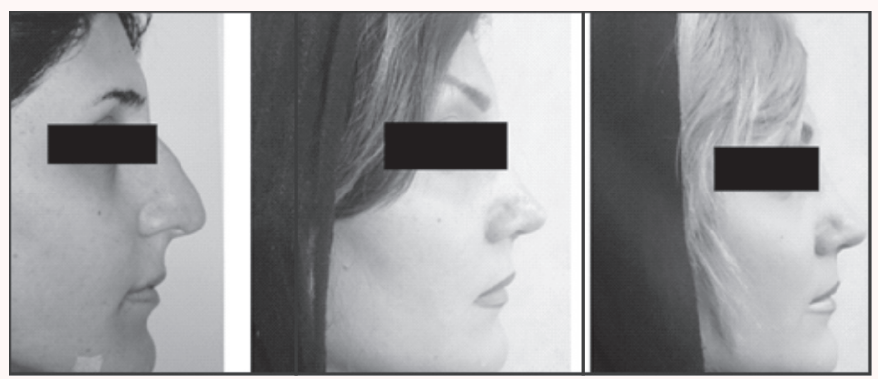

Figure-1: 3 assessment stages of a patient treated with the SEG technique.

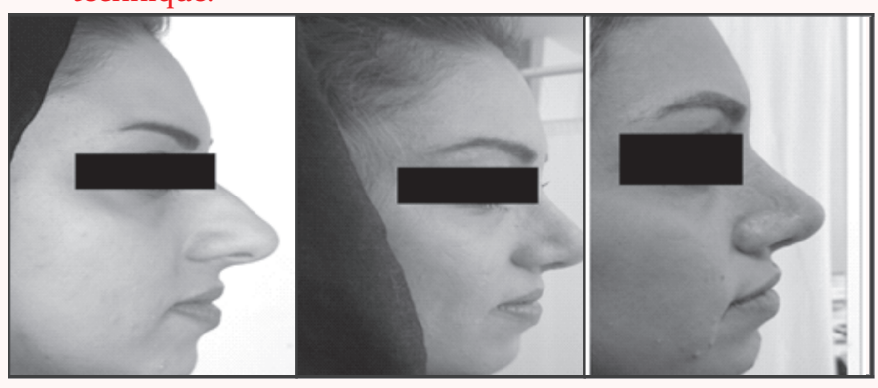

Figure-1: 3 assessment stages of a patient treated with the SEG technique.
In both the groups; male/female ratio was $2(15.88 \%) / 11(84.26 \%)$. The mean age \pm standard deviation (SD) in the SEG and the conventional group was $28.75 \pm 5.62$ and $29.58 \pm 6.82$, respectively and the difference was not statistically signif icant. There was no signif icant difference in the thickness of the pinched skin before and during operation in both groups ( $\mathrm{p}>0.05)$. Nasofacial angle was not significantly different between the two groups before and after surgery ( $\mathrm{p}>0.05)$, but after 3 and 9 months surgery, there was a signif icant decrease in SEG group.

Nasolabial Angle and columellar-labial angle before surgery were not significantly different between the two groups but revealed a significant increase in SEG group within 3 and 9 months after surgery. Nasocolumellarangle had no significant difference before and 3 months after surgery between the two groups, but 9 months after surgery in the SEG group was signif icantly higher.

Mean TP: LN ratio was not significantly different between two groups before and 3 months after surgery, but9 months after surgery, this ratio in the the group was signif icantly higher. The mean TP: LN ratio, 9 months after surgery in the conventional group was approximately equal to the preoperative level, but in the SEG group, it was higher than before surgery (Table-).

Table-1: Comparison of treatment outcomes in the two groups

\begin{tabular}{|c|c|c|c|}
\hline \multirow{2}{*}{\multicolumn{4}{|c|}{$\begin{array}{l}\text { Parameter- } \\
\text { The thickness of pinched skin }\end{array}$}} \\
\hline & & & \\
\hline Before surgery & $10.29 \pm 0.3$ & $6.4 \pm 0.3$ & 0.18 \\
\hline During surgery & $10.58 \pm 0.4$ & $5 \cdot 9 \pm 0.7$ & 0.22 \\
\hline \multicolumn{4}{|l|}{ Nasofacial Angle } \\
\hline Before surgery & $31.77 \pm 2.82$ & $31.87 \pm 2.11$ & 0.93 \\
\hline After 3 months & $28.48 \pm 4.10$ & $32.21 \pm 2.84$ & $0.04^{*}$ \\
\hline After 9 months & $29.42 \pm 2.65$ & $31.72 \pm 2.89$ & $0.04^{*}$ \\
\hline \multicolumn{4}{|l|}{ Nasolabial Angle } \\
\hline Before surgery & $90.41 \pm 9.40$ & $92.04 \pm 8.39$ & 0.07 \\
\hline After 3 months & $98.33 \pm 1.02$ & $96.63 \pm 4.40$ & $0.04^{*}$ \\
\hline After 9 months & $101.22 \pm 7.80$ & $96.80 \pm 4.03$ & $0.001^{*}$ \\
\hline \multicolumn{4}{|c|}{ NasoColumellar Angle } \\
\hline Before surgery & $30.38 \pm 5.02$ & $31.61 \pm 2.57$ & 0.53 \\
\hline After 3 months & $32.54 \pm 3.20$ & $32.34 \pm 1.91$ & 0.72 \\
\hline After 9 months & $33.25 \pm 3.93$ & $31.84 \pm 2.11$ & $0.03^{*}$ \\
\hline \multicolumn{4}{|c|}{ Columellar-Labial Angle } \\
\hline Before surgery & $96.26 \pm 9.69$ & $97.01 \pm 8.36$ & 0.5 \\
\hline After 3 months & $107.17 \pm 2.5^{2}$ & $100.67 \pm 0.89$ & $0.001^{*}$ \\
\hline After 9 months & $105.32 \pm 4.63$ & $94.43 \pm 3.34$ & $0.001^{*}$ \\
\hline \multicolumn{4}{|l|}{ TP:LN ratio } \\
\hline Before surgery & $0.62 \pm 0.01$ & $0.63 \pm 0.03$ & 0.11 \\
\hline After 3 months & $0.65 \pm 0.01$ & $0.66 \pm 0.04$ & 0.20 \\
\hline After 9 months & $0.64 \pm 0.01$ & $0.63 \pm 0.00$ & $0.01^{*}$ \\
\hline
\end{tabular}

\section{Discussion:}

In the present study, the nasofacial angle after 3 and 9 months of surgery was signif icantly lower in the SEG group. Nasolabial Angle and columellar-labial angle were signif icantly higher in the SEG group after 3 and 9 months 
surgery. Nasocolumellarangle was statistically higher in the SEG group 9 months after surgery. The mean TP: LN ratio at 9 months after surgery in the conventional group was approximately the same as before surgery, but in the SEG group, it was more than that before the operation and did not decrease after several months.

Benavides et al. (2019) compared the results of the caudal septal extension graft (CSEG) and the conventional method (fixation of the graft to the nasal spine region) with an average of 33.2 months of follow-up. They reported, tip deprojection, complication and reoperation rates were similar in both groups and did not show significant differences. Only the suture extrusion was less signif icant in the CSEG group. Mizuno (2019) studied a new technique for the use of hybrid autologous costal grafts with septal extension grafts in 28 patients. None of the patients experienced serious complications. The surgical results were reported as "very satisfied" in $42.9 \%$, "satisfied" in $35.7 \%$, and "required revision surgery" in $21.4 \%$ of cases. Using hybrid autologous costal grafts with septal extension grafts for augmentation rhinoplasty can achieve successful outcomes. Akkus et al. (2013) compared the effects of shortand long-term columellar strut and septal extension grafts in 36 patients. In both groups, nasal projection increased and was maintained in the long-term. Although there was no significant difference between the results of the two groups, the clinical and statistical findings showed that the ratios of nasal projection to nasal length and columellar labial angle values in the septal extension grafts group decreased significantly in the long-term.

Kim et al. (2014) examined the efficacy and disadvantages of three different types of septal extension grafts. During 6 months of follow-up, the tip angle and tip lengthening were significantly reduced in type II and the mean nasolabial angle in type III was significantly increased. The results of the study showed that according to patients' categorized and surgical objective, septal extension grafts could be achieved with sufficient nasal tip lengthening and projection.

In a retrospective study of Choi et al. (2014), the incidence of complications in the patients included infections $(4.5 \%)$, nasal tip stiffness (45.5\%), nasal tip deviation (11.4\%) and a decrease in projection (45.5\%). The results of the study showed that although the use of the SEG technique had acceptable results in terms of providing nasal tip support, projection, and length, the prevention of complications should be considered.

Finally, as per the results, it could be concluded that the septal extension technique by increasing and maintaining nasolabial, columellar-labial, and columellar-lobular angles, as well as a further reduction of the fissure nose angle and maintaining the TP: $\mathrm{LN}$ ratio over time, helps to maintain stability of projection and rotation of nasal tip, especially in patients with thick skin. Therefore, it seems that performing this technique in patients with thick skin has the greatest effect on maintaining the patient's augmentation.

Acknowledgment:

This study was conducted in Ahvaz JundishapurUniversity of Medical Sciences (AJUMS). The

study was financially supported by AJUMS (Grant.No.: U-98034)..

\section{References:}

Akkus, A.M., Eryilmaz, E. \& Guneren, E. (2013): Comparison of the effects of columellar strut and septal extension grafts for tip support in rhinoplasty. Aesthetic Plast. Surg., 37(4):666673.

Benavides, G., Villate, P. \& Malaver, C. (2019): Caudal eptal extension graft sutured with absorbable material and not fixed to the nasal spine region compared with the conventional fixation method: a retrospective study. Aesthetic Plast. Surg., 43(3):759-767.

Choi, J.Y., Kang, I.G., Javidnia, H. \& Sykes, J.M. (2014): Complications of septal extension grafts in Asian patients. LAMA Facial Plast. Surg., 16(3):169-175.

Chu, E. \& Davis, R.E. (2015): SMAS debulking for management of the thick-skinned nose. LAMA Facial Plast. Surg., 17(4):305306.

Cobo, R., Camacho, J.G., Orrego, J. (2018): Integrated management of the thick-skinned rhinoplasty patient. Facial Plastic Surg.,34(01):003-008.

Davis, R.E. \& Hrisomalos, E.N. (2018): Surgical Management of the Thick-Skinned Nose. Facial Plast. Surg., 34(o1):022028.

Katira, K. \& Guyuron, B. (2015): Contemporary techniques for effective nasal lengthening. Facial Plast. Surg. Clin. North Am., 23(1):81-91.

Khorasani, G., Hasani, O. \& Farahvash, M. (2010): Comparison of septocolumellar suture (SCS) and lateral crural overlay (LCO) methods on nasal tip projection and rotation in rhinoplasty. Tehran Univ. Med.J., 68(8):459-464.

Kim, J.H., Song, J.W., Park, S.W., Oh, W.S. \& Lee, J.H. (2014): Effective septal extension graft for Asian rhinoplasty. Arch. Plast. Surg., 41(1):3-11.

Kim, M.H., Choi, J.H., Kim, M.S., Kim, S.K. \& Lee, K.C. (2014): An introduction to the septal extension graft. Arch. Plast. Surg., 41(1):29-34.

Mizuno, T. (2019): A new technique for augmentation rhinoplasty using hybrid autologous grafts with septal extension grafts in asian patients. Facial Plast. Surg., 35( o1):58-64.

Wulkan, M. (2015): The Multi-lock system for rhinoplasty. Aesthetic Plast. Surg., 39(6):881-887. 